\title{
LA PERSONNE GRAMMATICALE EN INGOUCHE
}

Par Françoise GUERIN

Université Paris-IV Sorbonne, Paris

Laboratoire THEDEL Paris $V$, Paris

\begin{abstract}
:
This article presents a study on the "grammatical person" in ingush (a language of the North-Center Caucasus). It is carried out in various fields such as syntax, morphology and axiology. In order to anchor the analysis in a typological aiming, the author proposes a comparison with the expression of the person in abkhazian, language of the North-West Caucasus.
\end{abstract}

\section{INTRODUCTION :}

L'ingouche est une variante du tchétchène, langue de la famille caucasique, qui est parlée en Ingouchie, petite République fédérée à la Russie, située dans le Caucase nord centre. Ce pays est entouré au nord par la Russie, à l'est par la Tchétchénie, à l'ouest par l'Ossétie du Nord et enfin au sud par la Géorgie. Ce territoire de la taille d'un département français est peuplé de moins de deux mille habitants qui sont pour la plupart bilingues : russe-ingouche. L'ingouche, comme la majorité des langues parlées au Caucase, est considéré comme une langue minoritaire, et est cantonné depuis des décennies au rang de langue réservée au cercle familial et amical, jamais utilisé ni pour l'enseignement, les affaires économiques et administratives ni pour la justice. La transmission de l'ingouche a connu des interruptions assez longues au cours du $X^{\mathrm{e}}$ siècle et ce n'est que depuis la chute de l'Union Soviétique que les parents recommencent à apprendre l'ingouche aux enfants et que les tentatives gouvernementales pour redonner une place d'importance aux langues minoritaires se multiplient. Toutefois, rien pour l'instant n'a été mis concrètement en œuvre.

Les données exploitées dans cet article sont issues de corpus oraux enregistrés soit à Paris auprès de deux Ingouches entre 1995 et 1999 soit auprès d'une dizaine d'informateurs à Nazran capitale de l'Ingouchie en 1997. 


\section{QUELQUES POINTS IMPORTANTS DE SYNTAXE CONCERNANT L'INGOUCHE}

Cette langue présente une très nette opposition verbo-nominale, c'est-à-dire que lorsque l'on établit les compatibilités des unités de première articulation, on s'aperçoit qu'un grand nombre de monèmes est déterminé par des modalités (unités grammaticales déterminantes, jamais déterminées) qui leur sont propres et qu'un autre vaste ensemble de monèmes est, lui, déterminé par d'autres modalités. Ce constat permet donc de dégager une classe de verbes déterminée essentiellement par des modalités temporelles, aspectuelles et de voix et d'autre part une classe de noms déterminée par des modalités nominales spécifiques telles que le nombre et les adnominaux ${ }^{1}$.

Il n'existe pas en ingouche de modalités prédicatives, c'est-à-dire d'unités qui pourraient déterminer une unité nominale et une unité verbale lorsque celles-ci sont le noyau central de la phrase, ces unités, nominales et verbales, ayant par ailleurs des compatibilités avec des modalités qui leur seraient spécifiques. On peut dire qu'en ingouche lorsqu'un verbe apparaît dans une phrase il est toujours le noyau central de l'énoncé.

L'ingouche présente une construction ergative majoritaire c'est-àdire que dans le cas d'un noyau central verbal plurivalent (bi ou tri valent), l'expansion obligatoirement exprimée, qui donc est en relation d'implication réciproque avec le noyau central, assume la fonction sujet sans passer par l'intermédiaire d'un monème casuel indicateur de fonction, c'est la seule à pouvoir ainsi déterminer directement le noyau central. Elle se comporte de la même façon que l'expansion unique d'un verbe monovalent (à l'exception des verbes qui expriment la connaissance, les sentiments et les perceptions ${ }^{2}$ ). Sémantiquement, l'expansion qui actualise un verbe bivalent joue prototypiquement le rôle de patient et jamais le rôle d'agent. L'expansion qui joue le rôle d'agent assume une fonction spécifique qui en ingouche est indiquée par le monème casuel ergatif.

\footnotetext{
1 Je regroupe sous le terme d'adnominaux les unités grammaticales qui déterminent le nom en dehors du nombre. Je préfère cette terminologie à celle de « déterminants du nom » qui est trop vague et à celle d' «actualisateurs du nom » qui est ambiguë puisqu'un présentatif peut actualiser un nom et pourtant il n'appartient pas à la même classe.

2 L'expansion unique de ces verbes déterminera toujours le noyau central par le biais du monème indicateur de fonction datif.
} 


\section{INVENTAIRE DES MONEMES PERSONNELS GRAMMATICAUX}

En ingouche, comme je le présume dans la majorité des langues, les monèmes ou synthèmes qui se réfèrent à la personne sont nombreux et appartiennent selon l'étude de leurs compatibilités à diverses classes syntaxiques. La référence à un être humain peut en ingouche être donnée par une unité de la classe des noms (ex $d a$ «père »), par une unité de la classe des noms propres (ex : Aza, Para, Sultan qui sont des prénoms), une unité de la classe des cardinaux (ex. : tsa? «un ») et bien sûr par une unité de la classe des pronoms $(\mathrm{ex}:$ so $\ll \mathrm{je} »)$.

Seules les unités de cette dernière classe vont nous intéresser ici puisque ce sont elles que l'on appelle de façon générique «personne grammaticale », dénomination qu'il convient d'interpréter comme : unité linguistique à deux faces (signifiant/signifié) de première articulation ayant de façon directe ou indirecte une référence à la personne et formant une classe syntaxique particulière à inventaire restreint.

L'ingouche compte sept monèmes se référant à une personne déterminée, ce sont les unités suivantes : so « $\mathrm{P} 1 »$, $\hbar o \ll \mathrm{P} 2 », e z \ll \mathrm{P} 3$ éloigné », jer « $\mathrm{P} 3$ proche », vej « $\mathrm{P} 4$ inclusif », txo « $\mathrm{P} 4$ exclusif » et fo «P5» auxquels il faut ajouter les syntagmes constitués de la personne 3 proche déterminée par le pluriel: jer-e $\int$ \&.3 proche+pluriel » et la personne 3 éloignée déterminée par le pluriel : $e z-e \int$ « $\mathrm{P} 3$ éloigné+pluriel qui se contracte en $e \int 》$.

On totalise donc neuf monèmes qui représentent chacun une personne grammaticale définie par l'acte de communication. On peut ainsi dresser l'inventaire de ces unités.

Le paradigme des personnes grammaticales :

\begin{tabular}{|l|l|}
\hline P. 1 & so \\
\hline P. 2 & ho \\
\hline P. 4 (excl) & txo \\
\hline P. 4 (incl) & vej \\
\hline P. 5 & o \\
\hline & \\
\hline P. 3 (proche) & jer \\
\hline P. 3 (éloigné) & eS \\
\hline P. 3+ pluriel (proche) & jer-e $\int$ \\
\hline P. 3+ pluriel (éloigné) & e \\
\hline
\end{tabular}

Ce tableau nous enseigne que pour les Ingouches, le discours, l'acte de communication est plus largement centré sur le locuteur que 
sur l'auditeur puisque la personne 3 ne peut être localisée que par rapport au locuteur comme on le verra dans les lignes suivantes.

Comme dans toutes les langues, ces neuf monèmes de personne ont la particularité d'être des unités déictiques c'est-à-dire des unités qui ont comme toutes les unités de première articulation un signifiant et un signifié mais dont le référent est multiple, dépendant entièrement de l'acte de communication. Ainsi P. 1, en français par exemple, a pour signifiant /3ə/, pour signifié « locuteur » et pour référent Pierre, Paul ou Jacques selon la personne qui a pris la parole et ce même référent peut à tout instant devenir un auditeur, le /ty/ «l'auditeur » ou P. 2 puisque tout acte de communication présuppose un échange et donc un renversement des rôles : le locuteur devient l'auditeur et vice versa.

Chaque langue va, à partir des deux personnes qui fondent la communication, utiliser diverses stratégies pour désigner les divers participants à cet acte, l'ingouche dispose donc des éléments suivants :

- /so/ «P. $1 »$ : renvoie au locuteur, l'emploi de ce monème permet à celui qui parle de s'inscrire dans ce qu'il dit.

- / $\hbar$ / « P. 2 » : renvoie à l'auditeur. La deuxième personne réfère à l'individu qui participe à une situation de dialogue. C'est la personne à qui l'on parle par interpellation directe.

Lorsque le locuteur s'associe à l'auditeur pour exprimer en leur nom à tous les deux une opinion ou lorsqu'il s'adresse à plusieurs interlocuteurs, on observe assez fréquemment dans les langues l'emploi de la personne 1 ou de la personne 2 déterminée par le pluriel. Il est bien entendu que le pluriel utilisé n'est pas un vrai pluralisateur dans ce sens qu'il n'y a pas multiplication du même «je» ou du même «tu» mais multiplication de locuteurs ou d'interlocuteurs qui ne parlent que par l'intermédiaire d'une seule personne. D'autres langues, par contre, vont recourir à des formes spécifiques et c'est ce que l'on peut observer en ingouche, ainsi on dégage :

- |txo/ «P. 4 inclusive» : moi et toi ou moi et vous, renvoie à un ensemble de personnes qui comprend la ou les personnes à qui l'on parle et le locuteur.

- /vej/ «P. 4 exclusive » : moi et eux : ce monème exclut du groupe l'auditeur.

- / $\int \boldsymbol{o} / \ll \mathrm{P} .5 »$ : renvoie à un ensemble comprenant la personne à qui l'on parle plus une ou plusieurs autres personnes.

Ces cinq unités n'impliquent aucun référent lexical, ce sont de purs marqueurs de la personne c'est-à-dire qu'elles renvoient directement à une personne nécessairement présente dans l'acte de communication. 
C'est ce qui les distingue fondamentalement d'un point de vue sémantico-référentiel des personnes 3 .

Effectivement la personne 3 a plusieurs spécificités qui la démarquent des autres :

- Elle n'est pas instituée par la communication elle-même, elle est extérieure à l'interlocution.

- Elle peut renvoyer ou non à un humain ce qui est impossible pour les autres sauf si on se place dans une situation imaginaire

- Elle est soit anaphorique ou cataphorique et déictique, elle a toujours besoin de reprendre le référent d'une chose ou d'un être présent dans la situation communicative mais qui ne participe pas directement à l'échange. La phrase suivante en donne un exemple : il vient vers nous.

- Elle a, en ingouche, une forte valeur démonstrative. C'est une unité doublement déictique puisqu'elle intègre en plus une dimension spatiale. Elle fait partie d'un système à deux composantes qui évalue la distance qui sépare le locuteur du référent que désigne la troisième personne :

- /jer/ «P. 3 proche »: ne renvoie ni à celui qui parle ni à celui à qui l'on parle mais à un participant animé ou inanimé tout de même déterminé puisque proche du locuteur, dans sa sphère de vision et de contact.

- le $\int$ «P. 3 éloigné»: renvoie à un participant animé ou inanimé non défini, éloigné du locuteur ou absent au moment de la parole. Lorsqu'elle désigne une personne, celle-ci ne parle pas, ne reçoit pas d'interrogation directe et se trouve éloignée ou absente de la sphère de vision et de contact du locuteur.

D'un point de vue syntaxique, les personnes 3 présentent également des spécificités :

- Ce sont les seules unités du paradigme à pouvoir être, en ingouche, déterminées par le pluriel. C'est-à-dire que la pluralisation se réalise de façon mathématique, il y a bien multiplication d'une entité. Ce sont donc les seules à pouvoir se substituer à un nom (substantif) précédemment mentionné dans le discours.

- Enfin, elles sont les seules à avoir des compatibilités particulières qui devraient les exclure de la classe des pronoms : elles peuvent déterminer des noms :
$\begin{array}{llllll}\text { (1) } & \text { t'aq } & \text { ez } & \text { voaq } & \text { sek } & \text { ol } \\ \text { puis } & \text { P.3 él } & \text { vieux } & \text { homme } & \text { dit }\end{array}$
Puis ce vieil homme dit ....
ol 
Pour garder une cohérence au système et par souci didactique j'ai estimé qu'elles subissaient alors un transfert dans une autre classe appelée arbitrairement classe des adnominaux.

D'un point de vue typologique l'ingouche possède des monèmes de troisième personne intégrés au paradigme des personnels bien que leur comportement syntaxique et leurs valeurs propres les démarquent des autres personnes.

Il est important de souligner qu'en ingouche les monèmes personnels ne sont pas intégrés au syntagme verbal sous la forme d'indices personnels. La personne s'exprime donc toujours sous la forme d'un monème formellement libre, indépendant, autrement dit détaché du verbe lui-même.

\section{(2) so pariz-e vut \\ P.1 Paris+locatif va \\ «Je vais à Paris»}

Le verbe en tant que noyau central de l'énoncé reçoit évidemment un grand nombre de déterminations, et notamment celles des modalités temporelles, aspectuelles ou de voix. Il ne subit pas l'accord en nombre mais par contre, son signifiant est susceptible de varier. Effectivement si celui-ci commence par une voyelle alors le nom lui imposera un accord sous la forme d'un indice qui lui sera préfixé. Ces indices servent à indiquer à quelles catégories sémantiques, impossibles à définir aujourd'hui, les nominaux appartiennent (noms communs, noms propres et pronoms), ils se préfixent également et dans les mêmes conditions aux adjectifs. Il faut les considérer comme des signifiants discontinus du nom.

Lorsque le noyau central verbal est déterminé par différentes expansions nominales, c'est uniquement celle qui l'actualise directement (sans passer par l'intermédiaire d'un monème fonctionnel de type casuel) qui étendra son accord morphologique au verbe. Cela prouve son degré d'intimité et donc sa grande importance.

\section{LA PREDICATION}

La personne grammaticale ne peut pas être employée comme noyau central de l'énoncé.

\section{LES COMPATIBILITES}

La personne grammaticale détermine et peut être déterminée par les unités des classes syntaxiques suivantes : 


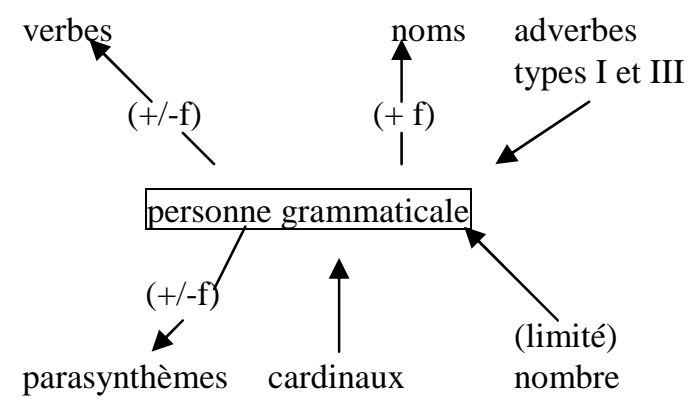

Ainsi la personne grammaticale détermine directement (ex. 3) ou par le biais d'un monème fonctionnel casuel (ex. 4) le noyau verbal central :

(3) $\quad \begin{array}{lll}\text { so } & \text { six } & \text { jut } \\ & P & \text { vite }\end{array}$

Je marche vite

(4) az xi : mol-er

P. 1/erg eau boit+passé

Je buvais de l'eau

Les personnes déterminent toujours les noms par l'intermédiaire d'un monème fonctionnel casuel (ex. 5) :

(5) se kine $\mathrm{k}$ istol-a kal ul

P. 1/gén livre table+sous est couché

«Mon livre est sous la table »

\section{LA MORPHOLOGIE DES PERSONNES GRAMMATICALES}

La morphologie est l'étude des variantes de signifiant que présentent les monèmes dans différents énoncés. Il convient également de prendre en compte tout ce qui ne fait pas l'objet d'un choix de la part du locuteur. Dès qu'il y a une contrainte, imposée par la langue, il n'y a pas pertinence communicative et donc ce luxe d'information et de forme est traité en morphologie.

La personne grammaticale subit en ingouche de nombreuses variantes de forme qui sont conditionnées par l'emploi d'un monème fonctionnel casuel.

Voici donc un nouveau tableau du paradigme des personnes grammaticales ingouches qui tient compte cette fois de toutes les variantes morphologiques dues à l'agglutination des monèmes casuels entraînant parfois des amalgames. 


\begin{tabular}{|l|l|l|l|l|l|l|}
\hline & & génitif & +datif & +destinatif & $\begin{array}{l}\text { /ergatif } \\
+\end{array}$ & +instrumental \\
\hline P. 1 & so & se & su-n & su-g & az & su-ts \\
\hline P. 2 & ho & he & hu-n & hu-g & ?a & hu-ts \\
\hline P. 4 (excl) & txo & txe & txu-n & txu-g & ox & txu-ts \\
\hline P. 4 (incl) & vej & vej & vej-n & vej-g & vej & vej-ts \\
\hline P. 5 & fo & fe & $\int_{\text {o-n }}$ & $\int$ u-g & o3 & Ju-ts \\
\hline & & & & & & \\
\hline P. 3 pr. & jer & uq & uqa-n & uqa-g & uq-u & uqan-ts \\
\hline P. 3 él. & ez & tse & tsi-n & tsin-g & ts-u & tsin-ts \\
\hline P.3 pro/plu & jer-e $\int$ & eqar & eqa-n & eqar-g & eqar & eqar-ts \\
\hline P. 3 él/plu & e & tsar & tsa-n & tsar-g & tsar & tsar-ts \\
\hline
\end{tabular}

L'ingouche présente, on l'a vu précédemment, la spécificité de catégoriser sémantiquement ses noms dans six groupes qui se répartissent en un genre ou sexe féminin, un genre ou sexe masculin et quatre genres neutres dont les distinctions sémantiques ne sont plus aujourd'hui perceptibles.

Ainsi que je l'ai déjà expliqué ces marques de genre apparaissent sous forme d'indice dans le verbe et dans l'adjectif sous réserve que ces derniers commencent par une voyelle. Il y a six groupements axiologiques dont certains présentent des syncrétismes de forme, les indices se répartissent comme ceci :

Genre ou sexe masculin :sg : v-, pl. : b-

Genre ou sexe féminin : sg : j-, pl. : b-

Catégorie sémantique $3: \mathrm{sg}: \mathrm{j}-, \mathrm{pl} . \mathrm{j}$ -

Catégorie sémantique $4: \mathrm{sg}$. : b-, pl. : d-

Catégorie sémantique $5: \mathrm{sg}$. : d-, pl. : d-

Catégorie sémantique $6: \mathrm{sg}$. : b-, pl. : b-

$\mathrm{Si}$ je mentionne de nouveau ce fait, c'est qu'il range l'ingouche dans la catégorie rare des langues qui indique le genre ou le sexe de la personne grammaticale. Le français, par exemple, distingue au niveau de la forme entre le genre ou sexe féminin et masculin, uniquement pour la personne 3, l'arabe le fait en plus pour le sexe de la personne 2. L'ingouche va exprimer le sexe ou le genre à toutes les personnes et c'est ce phénomène qui est peu fréquent. Ainsi, la personne 1 dira :

Si c'est un homme

(7)

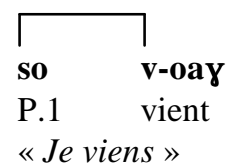


Si c'est une femme :

(8)

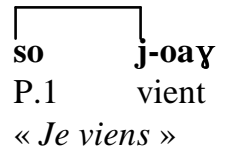

Si dans une fiction, c'est une poule qui parle :

(9)

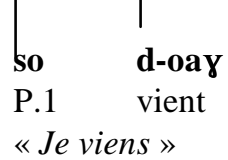

Si toujours dans une fiction, le cheval se met à parler, il dira :

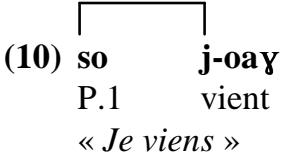

Si c'est un bélier qui s'exprime, il dira :
(11) so b-oay
P.1 vient
«e viens »

Si enfin c'est une souris :

(12)

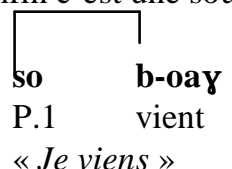

On observe une même forme d'indice dans les exemples (11) et (12) et pourtant le nom daxk «souris » appartient à un autre groupement sémantique que le nom kal «bélier», on s'en rend compte lorsque le nom est déterminé par le monème pluriel l'indice est alors distinct. Il en va de même pour les exemples (8) et (10) qalseg «femme » et govr «cheval» n'appartiennent pas au même groupement sémantique.

Cette particularité revient à montrer que le monème personnel en ingouche, tout comme le nom, est le plus souvent un monème à signifiant discontinu.

Il existe un deuxième paradigme des personnes grammaticales en ingouche lié à l'expression de la mise en valeur ou du réfléchi. Je les considère, d'un point de vue syntaxique, comme faisant partie de la même classe que les personnes grammaticales déjà citées, elles ont effectivement les mêmes compatibilités c'est pourquoi elles 
n'apparaissent qu'en morphologie. Ce sont des formes encore plus centrées sur la personne, elles permettent ainsi une certaine insistance sur une personne particulière.

\section{(13) sej kine $\int k$ jazdu \\ P.1/erg livre écrit}

«J'écris moi-même un livre »

Ce sont des personnels dérivés donc des synthèmes formés du monème de personne grammaticale suivi d'un suffixe d'insistance, on trouve donc :

$$
\begin{aligned}
& \text { /sej/ « moi-même », (P. 1) }
\end{aligned}
$$

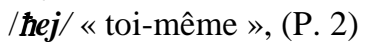

$$
\begin{aligned}
& \text { |txo } \mid / \text { « nous-mêmes » (exclusif), (P. } 4 \text { excl.) } \\
& / v o \int / \text { « nous-mêmes » (inclusif), (P. } 4 \text { incl.) } \\
& \text { / } \int \boldsymbol{o} \int / \text { « vous-mêmes », (P. 5) }
\end{aligned}
$$

Il paraît plus probable de concevoir que le suffixe /-j/ a subi après voyelle postérieure arrondie un changement phonétique plutôt que de considérer que le réfléchi 4 excl. par exemple se compose de $\mathrm{P} .4$ déterminé par le monème pluriel, cette vision des choses serait, à mon avis, un non sens.

Il est intéressant de noter qu'il n'y a plus de distinction de repérage spatial pour les réfléchis de troisième personne, on ne constate qu'une seule forme :

$/ \int i /$ « soi-même, lui-même », (P. 3)

/ $\int$ oa $\int /$ « eux-mêmes », (P. 3/pluriel)

Toutes les personnes emphatiques vont également présenter des variantes de forme dues une fois encore à la présence des monèmes casuels.

\section{LES FONCTIONS ASSUMEES}

\section{PAR LA PERSONNE GRAMMATICALE}

La personne grammaticale, dans la mesure où elle remplace un nom, va pouvoir assumer par rapport au noyau central verbal, les mêmes fonctions que celui-ci.

La fonction sujet, la fonction objet et la fonction objet second sont illustrées dans la phrase suivante :
(14) az
tsi-n kestkest
ez ol
P.1/erg P.3él+dat souvent
P.3él dit
«Je le lui dis souvent»

et qui se visualise comme ceci : 


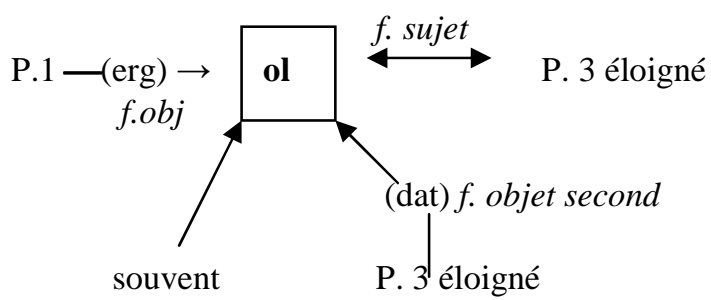

Les personnes grammaticales peuvent également assumer des fonctions non spécifiques telles que: la fonction instrumentale ${ }^{1}$ (ex. 15) tout comme la fonction circonstancielle (ex. 16).
(15) so vut ho-ts
P.1 va P.2+instrumental « Je vais avec toi »
(16) so hu-njixje lat
P.1 P.2+près de est debout
« Je suis debout près de toi »

\section{La MiSe EN VALEUR de La PERSONNE GRAMMATICALE}

Lorsqu'un Ingouche veut insister sur la participation de l'agent à l'action, il va ajouter les personnes emphatiques.
(17) es $\int_{i}$ voay
P. 3 él. P.3 vient
«Il vient lui-même »
(18) az ejs bjab jer berig bolx P. 1/ erg P. 1/erg fait/accompli P3. él. tout travail «J'ai fait moi-même tout ce travail »

La répétition de la personne grammaticale est également un procédé de mise en valeur.
(19)
hu-n hu-n
P. 2+dat P. 2+dat cerise
«A toi, il te donne la cerise »
lu tsu
donne P. 3 él/erg

\section{L'EXPRESSION DE LA POSSESSION}

Les personnes grammaticales peuvent indiquer le possesseur lorsqu'elles déterminent une autre unité par l'intermédiaire du monème fonctionnel génitif.

\footnotetext{
${ }^{1}$ La fonction instrumentale en ingouche a de multiples valeurs selon les unités mises en rapport.
} 
Certaines grammaires russes à l'instar de celle de Decheriev ${ }^{1}$ en font une classe à part en les considérant comme des adjectifs possessifs différents donc des pronoms personnels. Cette distinction est tout à fait superflue car elle ne tient pas compte du système pronominal et de ses compatibilités. Les traiter de «possessifs » c'est se référer uniquement à leurs traits sémantiques. Lorsqu'un nom détermine un autre nom par l'intermédiaire du génitif, personne ne pense alors à faire entrer ce dernier dans une classe nouvelle, celle des «noms possessifs $»^{2}$.

(20) he xatfi yand-a k'al ul

P. 2/gén pantalon chaise+sous est couché

Litt : « le pantalon de toi est sous la chaise »

«Ton pantalon est sous la chaise »
(21) as
tse majjen itsaj
P. 1/erg P. 3 él/gén voiture achète/accompli
«J'ai acheté sa voiture »

Pour insister sur l'appartenance, et ce uniquement lorsque l'objet possédé appartient à l'auteur de l'action, on aura soit un renforcement par la forme réfléchie, soit la forme réfléchie seule.
(22) $\mathrm{se}$

$\begin{array}{llllll}\text { se } & \text { sej } & \text { kine } \mathbf{k} \quad \mathbf{l u} & \mathbf{a z} & \text { hu-n } \\ \mathrm{P} .1 / \text { gén } & \text { P.1/gén livre donne } & \text { P. 1/erg } & \text { P. 2+dat } \\ \text { «Je te donne mon propre livre » } & & \end{array}$

\section{L'EXPRESSION DE L'INDEFINI}

L'absence de personne dans un énoncé comportant un verbe plurivalent, jouant le rôle sémantique d'agent donne une valeur indéfinie à la phrase. L'auteur ou les auteurs de l'action ne sont pas connus du locuteur ou celui-ci ne veut pas préciser leur identité. L'énoncé (23b) montre l'effacement de l'agent.
(23a) tsu
su-n djenet
je kine $\int \mathbf{k}$

P. 3/erg P. 1+dat donne/accompli P.3 él. livre

«Il m'a donné ce livre » ou «Ce livre m'a été donné par lui»
(23b) su-n
djenet
je
kine $\int k$
P. 1+dat donne/accompli P.3 él. livre
«On m'a donné ce livre » ou «Ce livre m'a été donné»

\footnotetext{
1 Decheriev, J. A., 1963, Grammaire historique comparative des langues nakhs, Livres Tchétchéno-ingouches, Grozny, 555 p. (Ouvrage publié en russe).

${ }^{2}$ Maria TSIGOU, Pronoms personnels et pronoms possessifs : une seule classe en grec moderne, Travaux du SELF VI, Laboratoire Théorie et description linguistique (THEDEL), Université René Descartes-Paris V, 1996-1997, p. 38.
} 


\section{COMPARAISON AVEC L'ABKHAZE}

Il m'a semblé intéressant de confronter l'ingouche à une autre langue caucasique d'un sous-groupe différent (tcherkesse occidental) mais de la même branche septentrionale afin de voir si la personne grammaticale y était ou non exprimée autrement.

Pour cette étude, j'ai utilisé des articles de Catherine Paris ${ }^{1}$ ainsi qu'un ouvrage de B. G. Hewitt ${ }^{2}$.

Si j'ai choisi cette langue, plutôt que le tcherkesse ou l'oubykh qui ont été également décrits par C. Paris, c'est parce que l'abkhaze présentait plus de similitude avec l'ingouche. L'abkhaze est parlé en Abkhazie, ancienne République Autonome de l'URSS, aujourd'hui rattachée à sa puissante voisine la Géorgie. Ce territoire autonome est situé au nord-ouest de la Géorgie, sur les rives de la Mer Noire. Ses locuteurs sont trilingues : russe, géorgien et abkhaze.

En abkhaze, et selon les données dont je dispose, la structure de la phrase est, comme en ingouche, majoritairement de type ergatif. Il semblerait que l'on dispose, dans cette langue, d'une opposition verbo-nominale et, là encore comme en ingouche, le verbe reçoit du nom, par accord morphologique, un indice.

L'abkhaze compte six monèmes personnels, mais seuls cinq sont toujours présents quel que soit le verbe exprimé : sə «P.1», wə «P. $2 »$, jə «P. 3 » ha «P.4 », s'ə «P. $5 »^{3}$.

On voit que toute la dimension spatiale qui était pertinente en ingouche pour la troisième personne n'existe plus en abkhaze, et qu'est absente également la distinction inclusif/exclusif au niveau de la personne 4. Il n'y a pas selon l'analyse des deux chercheurs de personne 6 avec les verbes monovalents, l'abkhaze utilise la personne trois, c'est donc le contexte ou la situation qui permettent à l'interlocuteur de savoir s'il y a ou non pluralisation de la troisième personne.

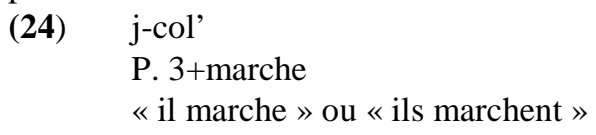

Par contre avec les verbes bivalents, il existe bien un sixième monème personnel qui se réalise sous la forme rə «P. 6 ».

\footnotetext{
1 Catherine PARIS, 1969, Indices personnels intraverbaux et syntaxe de la phrase minimale dans les langues du Caucase du Nord-Ouest, Bulletin de la Société de Linguistique de Paris, vol LXIV, fasc. 1, Paris, p. 104-183.

2 B. G. HEWITT, 1989, Abkhaz, London, New York, Ed. Routledge, 282 p.

3 C'est l'analyse proposée aussi bien par C. Paris que par B. G. Hewitt.
} 
A la différence de l'ingouche qui peut avoir pour énoncé minimum un nom actualisant un verbe, en abkhaze, les personnes grammaticales doivent nécessairement être exprimées, elles sont donc en imposition de coexistence ${ }^{1}$ avec le monème verbal, et apparaissent sous forme d'indices préfixés au verbe. Etant absolument nécessaire à la mise en œuvre de la langue elles actualisent le verbe et assument alors la fonction sujet. Le syntagme verbal forme ainsi un énoncé complet.

Il existe un paradigme de monèmes libres dit «emphatiques » servant notamment à l'expression de la mise en valeur de la personne grammaticale. Ce sont des synthèmes par composition formés de l'indice personnel auquel est ajouté un monème adverbial arà signifiant « ici $»^{2}$.

$$
\begin{aligned}
& \text { sarà } \quad \text { sə-col' } \\
& \text { P. } 1 \quad \text { P.1+marche } \\
& \text { «Moi, je marche » }
\end{aligned}
$$

Ces monèmes personnels emphatiques ne représentent pas une classe distincte.

Les variantes de signifiant au niveau du paradigme des indices personnels sont peu nombreuses car à la différence de l'ingouche, l'abkhaze ne fait pas appel aux monèmes casuels pour indiquer la détermination du noyau central verbal par les personnes.

On constatera toutefois une variante de forme pour la personne 2 ainsi que pour la personne 3. Effectivement, si l'ingouche présente un accord en genre ou sexe pour toutes les personnes grammaticales, l'abkhaze en revanche ne le fait que pour deux personnes.

La distinction s'établit pour la personne deux entre le genre ou sexe masculin et le genre ou sexe féminin. On aura donc soit wə «P.2 masculin »soit $b$ ə «P. 2 féminin ». Au niveau de la personne 3 c'est l'opposition raisonnable/non raisonnable ${ }^{3}$ qui est mise en avant avec les verbes monovalents alors qu'avec les verbes bivalents on observe une tripartition entre masculin, féminin et non raisonnable. On aura donc dans le premier cas : soit $d ə$ «P.3 raisonnable», soit jə «P. 3

\footnotetext{
1 La notion d'imposition de coexistence, proposée par Christos CLAIRIS, 1999, «Soulevons le lièvre... », Actes du XXIIIème colloque international de Linguistique Fonctionnelle, Lugano (à paraître), est conçue comme « la caractéristique de certains monèmes, ou de certaines classes de monèmes, d'impliquer la présence d'une ou plusieurs déterminations grammaticales pour qu'ils puissent fonctionner dans le discours. »

2 C'est l'explication donnée par B. G. Hewitt p. 46.

3 L'opposition raisonnable/non raisonnable fait référence à la dichotomie : doué de parole/non doué de parole. Ainsi l'enfant dans de nombreuses langues caucasiques relève de la catégorie des non raisonnables car il ne maîtrise pas encore parfaitement le langage, il est donc rangé dans la même catégorie que les animaux ou les choses par exemple qui eux n'ont pas accès au langage.
} 
non raisonnable » et dans le deuxième cas : jə «P.3 masculin », lə «P. 3 féminin » et $n a$ ou $a$ « P. 3 non raisonnable».

On obtient le tableau suivant :

\begin{tabular}{|l|l|}
\hline Signifiés & Signifiants \\
\hline P.1 & $\mathrm{s}$ \\
\hline P. 2 & $\mathrm{w}$ (masc)/b (fém) \\
\hline P. 3 & $\begin{array}{l}\mathrm{d} \text { (rais.)/j (non rais.) } \\
\mathrm{j} \text { (masc), l (fém), na/a (n rais) }\end{array}$ \\
\hline P. 4 & $\mathrm{ha}$ \\
\hline P. 5 & $\mathrm{~s}^{\circ}$ \\
\hline & $\begin{array}{l}\text { (j) } \\
\text { P. } 6\end{array}$ \\
\hline
\end{tabular}

L'originalité de l'abkhaze sur le plan de la personne grammaticale réside dans son traitement syntaxique. Le syntagme verbal va contenir toutes les personnes qui participent d'une façon ou d'une autre à l'accomplissement du processus or toutes les personnes sauf la 3 et la 6 auront toujours la même forme quelle que soit la fonction qu'elles assument au sein du syntagme verbal.

La position des unités dans le syntagme va donc être capitale pour comprendre le rôle de chacun.

Les verbes monovalents n'ayant qu'une expansion obligatoire, c'est l'antéposition au verbe qui est privilégiée :
(26) sə-col'
P.1+ marche
wə-col'
bə-col'
P.2 (masc)+marche
P.2 (fém)+marche
«e marche»
«tu marches»
«tu marches»
də-col'
jə-col'
P. 3(raison.)+marche
«Il marche»
P.3 (non raison.)+marche
«il marche » ou «ils marchent »

$\begin{array}{ll}\text { ha-col' } & \mathbf{s}^{\circ} \boldsymbol{\theta} \text {-col' } \\ \text { P.4+marche } & \text { P.5+marche } \\ \text { «Nous marchons » } & \text { «vous marchez » }\end{array}$

Les personnes grammaticales assument la fonction sujet et jouent des rôles divers, agent, expérient, possesseur, selon le sens du verbe.

Les verbes bivalents régissent deux expansions, l'expansion qui se comporte en tous points comme l'expansion unique d'un verbe monovalent va se trouver à la première place dans l'antéposition c'està-dire la plus éloignée du verbe. 
(28) jə-sə-swajt'

P.3 (n rais. )+P. $1+$ frappe

«Il me frappe »ou «ils me frappent »

Par l'analyse, on constate que l'expansion qui se trouve en position 1 joue le rôle de l'agent tandis que la deuxième expansion (la plus proche du verbe) assume la fonction objet et joue le rôle de patient.

Mais, on constate que tous les verbes bivalents ne fonctionnent pas de la même façon, ainsi :

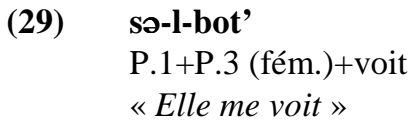

Dans cet exemple, on observe que l'expansion en position 1 (la plus éloignée du verbe) se réfère cette fois au patient et que l'expansion la plus proche du verbe joue le rôle de l'expérient c'est-àdire d'une sorte d'agent moins volontaire mais pas complètement patient.

Avec les verbes trivalents, l'expansion 1 va se référer au patient, l'expansion 2 au bénéficiaire et l'expansion 3 (la plus proche du verbe) joue le rôle de l'agent.

(30)

$$
\begin{aligned}
& \text { j-lo-s-tot' } \\
& \text { P.3 (n.rais)+P.3 (fém)+P.1+donne } \\
& \text { «Je le donne à elle » }
\end{aligned}
$$

S'il parait évident que la personne grammaticale en abkhaze peut assumer envers le noyau verbal les trois grandes fonctions: sujet, objet et objet second, je n'ai pas assez de données pour savoir avec certitude quels rôles le monème personnel en fonction sujet joue selon les verbes exprimés.

Ce qui est directement observable et ce qui est confirmé par l'analyse des deux linguistes spécialistes de cette langue, c'est que c'est la valence du verbe et les rôles qu'il implique qui vont déterminer la position des expansions dans le syntagme verbal :

- Verbes monovalents :

Expansion personnelle (rôles divers) verbe

- Verbes bivalents A :

Expansion 1 (agent), expansion 2 (patient) verbe 
- Verbes bivalents B :

Expansion 1 (patient), expansion 2 (expérient) verbe

- Verbes trivalents :

Exp. 1 (patient), exp. 2 (bénéficiaire), exp. 3 (agent) verbe

Les deux auteurs s'accordent pour indiquer que la position 1 est la position la plus importante, il semblerait donc que pour les verbes plurivalents, il y ait un changement d'orientation du verbe lorsqu'on se trouve face à des verbes bivalents de la catégorie A. Pour ces verbes-là, il convient de privilégier l'agent alors que pour les bivalents de catégorie B et les trivalents c'est le patient qui est important prouvant de cette façon leur structure ergative.

Si l'ingouche a un traitement clair de la personne grammaticale, il est plus difficile de concevoir une langue qui, comme l'abkhaze, n'a pas de marque formelle pour distinguer les personnes selon la fonction qu'elles assument et qui n'indiquent pas forcément par la position le rôle qu'elles jouent dans l'énoncé. C'est le sens du verbe et sa valence qui seuls donnent la clé du jeu de la personne dans et hors du syntagme verbal.

La non transmission mais surtout le fait que cette langue a été étudiée au sein de la diaspora, explique peut-être cet étrange phénomène que l'on retrouve dans les langues sœurs décrites par C. Paris ; le tcherkesse parlé en Turquie et l'oubykh aujourd'hui disparu. Peut-être que ce manque de repère dans les fonctions qu'assument les personnes grammaticales pourrait être interprété comme un signe annonciateur de la mort de la langue au sein des communautés abkhazes émigrées. 